\title{
Eccentric head positions reveal disorders of conjugate eye movement
}

\author{
M. G R E S T Y \\ From the Medical Research Council Hearing and Balance Unit, Institute of Neurology, \\ National Hospital, Queen Square, London
}

SUMMARY The effect of head position on conjugate horizontal gaze was studied in healthy adults, in patients with multiple sclerosis without eye movement signs, and in patients with downbeat nystagmus indicative of low brain stem lesions. Displacements of gaze from primary position to $30^{\circ}$ left and right were recorded using the electro-oculogram, with the head in the primary position, and turned voluntarily to the left and right (in yaw). The quality of eye movements was noted and peak velocities of saccades were measured. The head turning test trebled the incidence of abnormal eye movements found in the multiple sclerosis patients and increased it by tenfold in the patients with downbeat nystagmus. Disorders of eye movement were also found in approximately $20-30 \%$ of healthy subjects tested. Weakness of abduction was the most common eye movement defect and appeared to be posterior internuclear ophthalmoplegia. A0 hypothesis is made which unifies the theoretical explanations of anterior and posterior inter-零 nuclear ophthalmoplegia. The most likely cause of the disorders of eye movement observed is vertebrobasilar ischaemia induced by stretching and compression of the vertebral arteries during eccentric head posture.

When the head is turned from its primary position several things happen which have effects upon activity within the central nervous system.

Firstly, if the head rotates in the horizontal plane (yaw) so that the chin points to the right or left, the cervical spinal cord and the lower brain stem are deformed in torsion and shear, and this may be accompanied by compression of one side and stretching of the opposite side. The mechanical distortion of the brain stem probably reaches as high as the level of the tentorium cerebelli, and undoubtedly affects the cerebellar peduncles and at least part of the body of the cerebellum (personal observations on the brains of cats and monkeys fixed with the head held in different positions).

Secondly, displacement of the head in yaw produces differential movements between the foramen magnum, the atlas, and the axis which lead to compression of the vertebral artery on the side towards which the head is turned and narrowing of the artery on the opposite side (De Kleyn and Nieuwenhuyse, 1927; De Kleyn, 1939; Tissington

Accepted 26 May 1977 and Bammer, 1957). The mechanical effects of the spinal arteries may be also relevant. As a consequence of head turning local circulatory changes arise from neck muscle contraction and from stretching, compression, and decompression effects on arteries, arterial baroreceptors, and veins. Normally, compensations are made for the changes in circulation induced by head turning so that adequate circulation is maintained. However, in the presence of anatomical anomalies such as abnormally narrow arteries, or pathological changes such as osteophytes which may deform blood vessels, head turning can produce insufficiencies of cerebral blood flow.

Thirdly, head posture gives rise to a pattern of proprioceptive signals from muscle and joint receptors in the neck which have an important influence on sensorimotor coordination (Longet, 1845; Magnus, 1924; Cohen, 1961).

The medulla, pons, and cerebellum contain motor and important premotor centres for the control of eye movements. Lesions in these centres can give rise to disorders of eye movements which can be revealed by appropriate test procedures. It may be that the mechanical and vascular changes 
produced by head turning can heighten the degree of oculomotor impairment and reveal latent defects not detectable with the head in its primary position. To investigate this hypothesis we have compared displacements of horizontal gaze with the head in its primary position with those executed when the head was turned to eccentric positions, both in a group of normal subjects and in patients with a variety of diseases of the nervous system.

Saccadic velocities and conjugation of gaze were selected for examination to minimise the involvement of postural reflex effects on eye movements. Vascular derangements and mechanical distortion remained as the main variables. Although postural (particularly neck) mechanisms are known to affect eye movements in normal subjects (Meiry, 1971) and in patients with nervous system diseases (Gray, 1956; Bos, 1962; Bos and Philipszoon, 1963; Biemond and De Jong, 1969), it has not been demonstrated that they influence either saccadic velocities or the organisation of conjugate horizontal gaze.

Neurological testing with the head turned is not new. The procedure has been used extensively in patients with cerebrovascular symptoms implicating abnormalities of the vertebral arteries (Biemond, 1951; Ford, 1952; Tissington and Bammer, 1957; Sheehan et al., 1960). It has been found that head turning reveals a wide variety of symptoms indicative of vertebrobasilar ischaemia. However, we know of no reports of the test being used comparatively in normal subjects and the types of patients we have surveyed, no attempts to quantify the results of head turning, and no studies of its effects upon conjugate gaze.

\section{Method}

PATIENTS With NEUROLOGICAL DiSEASES

Two groups of patients with central nervous system diseases were examined. One consisted of five patients between the ages 19 and 30 years who had multiple sclerosis with no clinically observed ocular signs. All were part of a longitudinal survey conducted at the National Hospital and had been observed for several years.

The second group of patients was selected because they presented with the oculomotor sign of 'downbeat nystagmus'. Downbeating nystagmus is taken to be a reliable sign of lesions of the lower brain stem and structural disorders in the region of the foramen magnum (Cogan, 1968). These regions of the nervous system are the most likely to be affected by mechanical or vascular concomitants of head turning.
CONTROL GROUPS

The selection of normal subjects for control groups presented a problem because it required a definition of normality. Several authors have described the eye movements of 'normal' subjects during displacements of horizontal gaze (Vossius, 1960; Smith et al., 1970; Weber and Daroff, 1971; Bird and Leech, 1976), and all agree that there are wide variations of accuracy, velocity, convergence, and conjugation of movement. We collected 15 subjects for controls and found that in four of these the head turning test revealed eye movements which appeared to be deranged. The problem was to decide whether the apparent derangements were simply unusual in that they represented the extremes of statistical distribution of eye movement characteristics, or whether the eye movements were abnormal in the sense of pathological. This decision was made with reference to several criteria. The first and most important criterion for an abnormal saccade was a consistently low peak velocity. The second criterion was that the shape of the saccade was consistently degraded on repeated testing (compare, for example, the normal and abnormal saccades in Figs. 2 and 3). In addition, when saccades were made which appeared abnormal it was sometimes found that other signs of oculomotor impairment, such as nystagmus, were also present. The velocity and shape of a particular saccadic movement were compared with those of the same movement in different head positions, with those of the other subjects tested, and with the data of other workers. If two or more of the above criteria applied to a saccadic eye movement, the movement was judged to be abnormal and the subject was excluded from the control groups. Examples of abnormal movements found in 'normal' subjects are presented in group 2 of the results.

Two control groups with five subjects in each were set up, divided into the age ranges of 19-31 and 50-65 years respectively. The latter group can be expected to include a higher incidence of vascular disorders. The subjects in the control groups exhibited a wide range of saccade velocities; however, the saccades they executed were consistently well formed and unaffected by head turning.

\section{TESTING PROCEDURE}

The subjects were required to execute saccadic eye movements between a target in primary gaze and targets $30^{\circ}$ to the left and right, placed at a distance of $700 \mathrm{~mm}$ with the head in the primary position and turned voluntarily to the left and right (illustrated in Fig. 1). Because of problems 




Fig. 1 Illustration of apparatus used to test saccadic eye movements. Subject makes eye movements to fixate targets on horizontal bar with head held voluntarily in three positions. The bar is supported by the subject and stabilised with a chin rest.

of head mobility in some patients, predetermined angular displacements of the head could not be achieved. Instead, subjects were asked to turn their heads to the most eccentric positions they could comfortably maintain. Displacements greater than $45^{\circ}$ were achieved in all subjects tested, care being taken to ensure that when the head was turned its sagittal plane remained vertical.

Eye movements were recorded using DC electrooculography, measurements of peak saccadic velocities being made with a nomograph and restricted to saccades which were free from blink and myogenic artefacts. As a result there were limitations on the number of measurements available.

\section{Results}

\section{TESTING SIGNIFICANCE OF EYE MOVEMENT}

\section{ABNORMALITIES}

In both patients and control subjects there were large interindividual differences in saccade velocities, and the data from each individual had high variances. When disorders were revealed by head turning they followed idiosyncratic patterns which could not be easily tested against the normal controls. Accordingly, three criteria were chosen to test the significance of the findings.

1. The lower level of mean saccadic velocity for healthy eye movements was set at $320^{\circ}$ second. This is a low value for any of our normal subjects and gives a probability level of less than $2 \%$. It is also well below the criterion for normality set by Bird and Leech (1976) determined in the same laboratory.

2. The patterns of changes of eye movement as a function of head position were also taken into account in each individual.

3. The statistics for a given eye movement and head position were compared with those of the same movement in the other positions in order to detect trends.

GROUP 1 SACCADIC MEASUREMENTS OF NORMAI SUBJECTS

No degrading effects of head turning on conjugate horizontal gaze were found in normal subjectsThe findings are in agreement with other authors (Bird and Leech, 1976) who tested only in heaô primary position, in so far as abduction was slower than adduction and decentring was slower than centring. The younger group had slightly higher mean velocities than the older controls who exhibited higher variances. The data are presented in Table 1 for subjects in the age ranges 19-31 and 50-65 years.

GROUP 2 ABNORMAL EYE MOVEMENTS REVEALED IN NORMAL SUBJECTS BY HEAD TURNING

In approximately $23 \%$ of the normal subjects tested, the head turning test revealed derangements of ocular motility. The derangements were either multiple, constituting a syndrome, or a single defect was revealed. These observations will be described in three subjects whose saccade metrics are detailed in Table 2.

Subject 1-male, 56 years old. In all head positions a right eye decentring and left eye centring abduction weakness were present. With head turned to the right a first degree nystagmus was present in the right eye, and in the same eye abduction was badly formed and took a long time to settle in the eccentric gaze position. 

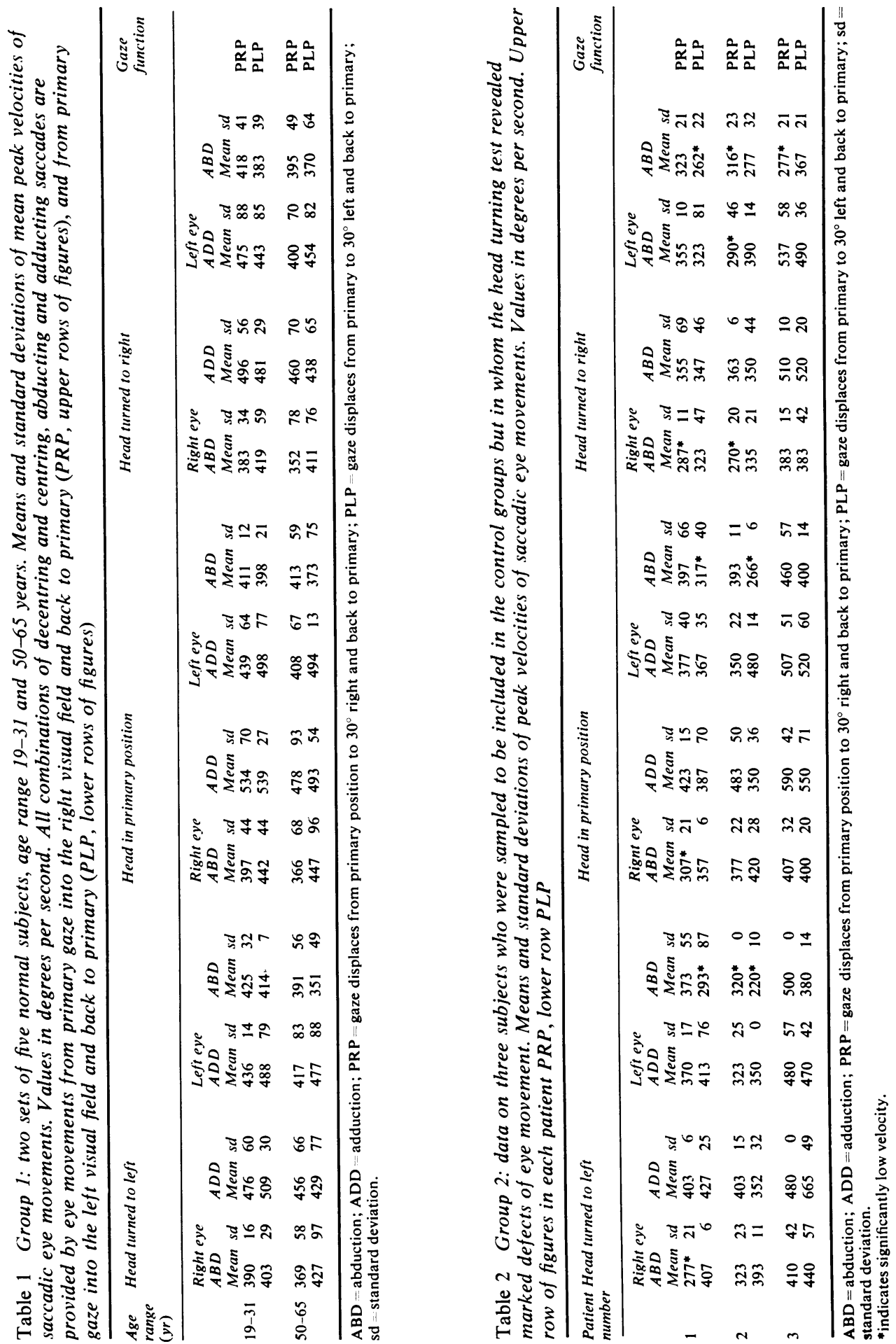
Subject 2-female, 25 years old. With the head in primary position there was a weakness of the left eye in centring abduction movements; this was exacerbated with head turned to the left. With head turned to the right, the right eye developed a decentring abduction weakness and the left eye, a further adduction weakness (Fig. 2). With head turned to the right there was also an observable first degree nystagmus of the right eye which rebounded.


Fig. 2 Decentring and centring eye movements executed between primary gaze $(P)$ and a target $30^{\circ}$ right. Subject 2 of group 2. $R=$ right eye; $L=$ left eye; arrowheads-saccades of nystagmus. With head rotated to right the right eye makes an abnormal abduction movement, which can be compared with the normal abduction made by the right eye in the head primary position, and has a first degree nystagmus.

Subject 3-female, 22 years old. Eye movements in all head positions were within the normal range with one exception. With head turned to the right there was a centring abduction weakness of the left eye (Fig. 3). This finding was repeatable, and the low velocity of the saccade was statistically significant.

GROUP 3 EFFECTS OF HEAD TURNING ON SACCADIC EYE MOVEMENTS IN MULTIPLE SCLEROSIS

Measurements of peak velocities of saccadic eye movements are presented in Table 3 . The table shows that patients 2 and 5 had weakness of saccadic eye movement when tested with the head in its primary position. Four patients had weakness
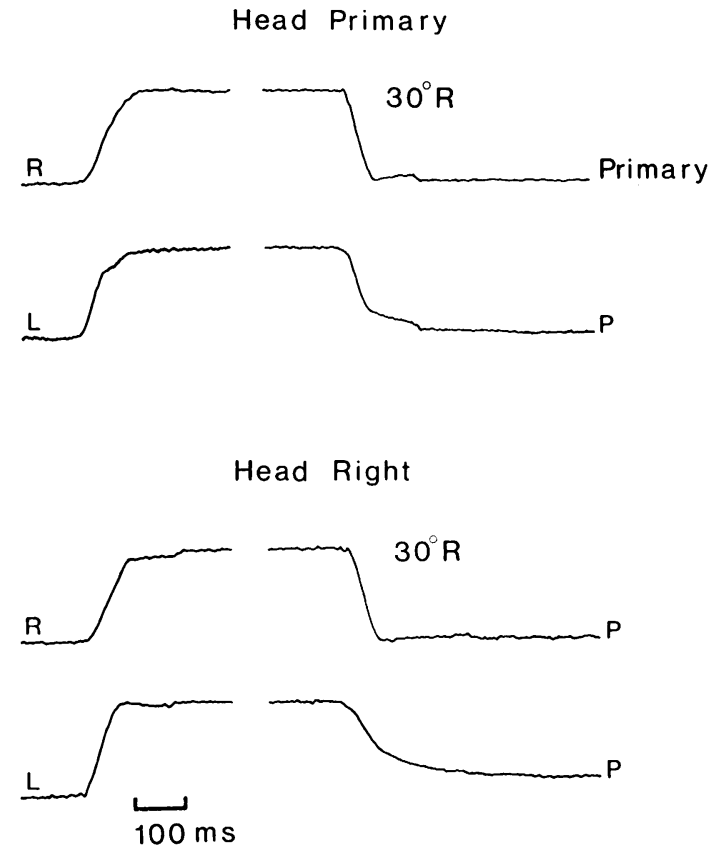

Fig. 3 Decentring and centring eye movements executed between primary gaze $(P)$ and a target $30^{\circ}$ right. Subject 3 of group 2. $R=$ right eye; $L=$ left eye. The left eye has a weakness of centring abduction in the head right position. All other saccades are normal.

of saccadic movements when the head was turned laterally. Head turning had no effect on one patient. This disorder revealed by head turning in these patients was predominantly a weakness of abduction.

The head turning test doubled the incidence of detection of abnormal saccades in this group of patients. It is noteworthy that no evidence of anterior internuclear ophthalmoplegia was revealed by the test.

GROUP 4 DISORDERS OF EYE MOVEMENT REVEALED BY HEAD TURNING IN PATIENTS WITH DOWNBEAT NYSTAGMUS

Four patients presenting in common the symptom of downbeat nystagmus and suffering from a variety of neurological diseases were selected for this study. The statistics relating to peak saccadic velocities are presented in Table 4 . In all patients, head turning revealed further defects of eye movement. Again, the pattern of disorder revealed by head turning has to be considered in respect of each individual patient and no generalisations can be made. Head turning showed weakness of abduction or adduction in almost equal numbers. 










Clinical observation had revealed no disconjugation of eye movement in these patients.

Patient 1, a 36 year old female, presented with a predominantly left sided cerebellar syndrome and brain stem signs. Neurological examination revealed gait ataxia, increased tone in all four limbs, and exaggerated reflexes. Tomograms of the foramen magnum showed backward deformity of the odontoid process encroaching upon the foramen magnum. There was an anomaly of the arch of the atlas. A myelogram showed signs of cervical syringomyelia with cerebellar ectopia associated with arachnoiditis and vascular proliferation.

Saccadic eye movements:

Head primary-shape and velocity were found to be within normal limits.

Head turned to left - there was bilateral weakness of adduction and abduction.

Head turned to right-there was adduction weakness of the right eye and adduction and abduction weaknesses of the left.

Patient 2, a 73 year old female, presented with predominantly left sided cerebellar signs, poor convergence, and impaired vibration sense. The lesions were probably widespread and vascular in nature.

Saccadic eye movements:

Head primary - the shape and velocity were found to be within normal limits.

Head turned to left-there was bilateral weakness of abduction.

Head turned to right-movements were within normal limits.

Patient 3, a 39 year old male, presented with multiple brain stem and cerebellar signs in the form of gait ataxia and weakness of the legs, exaggerated tendon jerks, extensor plantar responses, and clumsiness of the right hand. Necropsy showed an Arnold Chiari type 1 malformation. The right cerebellar tonsil extended across the midline and was larger than the left; they extended caudally to $15 \mathrm{~mm}$ below the level of the arch of the axis. The medulla oblongata was elongated.

Saccadic eye movements:

Head primary-abduction weakness was present in the left eye.

Head turned to left-there was abduction and adduction weakness in the right eye.

Head turned to right-there was abduction weakness and improved left eye abduction.

Head turning to the left revealed right eye weakness and improved left eye abduction.

Patient 4, a 45 year old male, presented with a cerebellovestibular syndrome of seven years' duration believed to be familial cerebellar degeneration. (The earliest sign to appear was downbeat nystagmus).

Saccadic eye movements:

Head primary-bilateral abduction weakness was present. The left eye adduction was weak.

Head turned to left-there was bilateral abduction and adduction weakness.

Head turned to right-there was bilateral abduction and adduction weakness.

COMMENT UPON SIGNIFICANCE OF TEST

On the sole basis of measurements of peak saccadic velocity, taking mean velocities of less than $320^{\circ} / \mathrm{s}$ as significant, head turning quadrupled the incidence of abduction weakness and increased the incidence of adduction weakness tenfold.

\section{Discussion}

IMPLICATIONS OF FINDING LATENT DISORDERS OF EYE MOVEMENT IN NORMAL SUBJECTS

The study demonstrates clearly that subjects with no neurological complaints can be shown to have 'latent' disorders of eye movement. There are two interpretations of this finding. Either the disorders merely represent the extremes of the normal range of behaviour or they are subclinical evidence of pathological lesions of the nervous system. The latter interpretation is favoured for several reasons. The abnormal eye movements revealed by head turning were not variations of the normal patterns but were often quite different in shape and associated with distinctly abnormal signs such as unilateral nystagmus. Furthermore, an abnormality of movement could be revealed in a specific head position; the same movement in the other two head positions was normal, and the characteristics of the movements did not appear to lie on the same statistical continuum. Finally, the peak velocity criterion adopted for the experiment was deliberately set very low, much lower than the criterion used for clinical testing in our own and in many other ENT laboratories.

If the conclusion is true that the test reveals lesions of the nervous system, it may be a sensitive indicator of susceptibility to vascular or degenerative disease.

POSSIBLE MECHANISMS BY WHICH HEAD TURNING REVEALS EYE MOVEMENT DISORDERS

Mechanical deformation of the brain or its vascular supply may alter the activity of nervous tissue by reducing local blood supply, thereby rendering the tissue anoxic. In addition, it is pos- 
sible that stretching or compression of nervous tissue can change the electrical characteristics of action potentials, rendering saltatory conduction less secure, in a way similar to the effect of heat on demyelinated peripheral nerve (Rasminsky, 1973). Either or both of these mechanisms may be operating in subjects with latent disorders of eye movement.

The patterns of abnormal movement revealed in normal subjects show most clearly how these mechanisms might operate. Subject 2 of group 2 showed several abnormalities of eye movement when the head was turned to the right. These included weak abduction of the right eye, adduction weakness of the left and a rebound nystagmus on right gaze. All these abnormalities are explicable if one assumes a degraded function of the right side of the brain stem and cerebellum. The degradation could arise if head turning to the right restricted flow through the right vertebral artery without adequate compensation from other blood vessels. For this state to occur it must be assumed that this person has vascular abnormalities.

In contrast, subject 3 of group 2 exhibited an isolated weakness of centring abduction in only one head position. To explain this it must be assumed that in the locality of the left abducens nucleus or of the neurones which inhibit the ipsilateral medial rectus motor neurones, local mechanical forces either restrict the vascular supply to these neurones or change their transmission characteristics. Again, an inherent defect is implicated.

In patients with multiple sclerosis there is the obvious possibility that mechanical deformation reveals functional defects in nervous tissue which is susceptible to stress because of demyelination. McAlpine et al. (1972) imply that head turning may be used to differentiate between multiple sclerosis and vertebrobasilar ischaemia because the head turning exacerbates the symptoms by increasing the extent of the ischaemia. However, ocular defects were revealed by head turning in the group of young people with multiple sclerosis in whom there was no evidence or likelihood of a vascular disorder. Our conclusion is that head turning can exacerbate disorders of oculomotor function in both multiple sclerosis and vascular diseases but is not an efficient test for differential diagnosis between them.

Patients with malformations in the region of the foramen magnum are most likely to have abnormalities of eye movement produced by vascular derangement because of the experimental demon- stration that, in injuries which compress structures passing through the foramen magnum, the vasculature is first, and nervous tissue last, to suffer (Taylor and Byrnes, 1974).

We have no convincing explanation for abnormal eye movements produced by head turning in patients with cerebellar degeneration. Such a patient reported in this study (patient 4 , group 4) had a massive loss of cerebellar volume. This could allow unusually large movements of the brain stem during head turning. However, the test is now used routinely in our laboratory, and recently it has revealed abnormalities of conjugate gaze in a patient with minimal atrophy of the posterior vermis of the cerebellum.

\section{NERVOUS MECHANISMS OF DISCONJUGATE EYE} MOVEMENTS

The most common defect this study encountered was an abduction weakness, even in the group of patients with multiple sclerosis. In particular, centring abduction was often found to be weak when decentring was normal. The abducens nucleus being the most caudally placed of all oculomotor nuclei is most likely to be affected by twisting the brain stem which would explain the high incidence of abducens weakness.

Abduction may be weak because firing rates in the sixth cranial nerve are low; alternatively, an apparent weakness may be seen because the ipsilateral medial rectus is not fully relaxed. This could result from a reduction of the inhibitory input to medial rectus motor neurones, which is applied during abduction (Maeda et al., 1972). It is believed that the inhibitory pathway to the ipsilateral medial rectus originates from about the level of the abducens nucleus and travels in the contralateral medial longitudinal fasciculus (Pola and Robinson, 1976) and, furthermore, that in the vicinity of the abducens nucleus are neurones which are responsible for conjugate lateral gaze (para-abducens nucleus). Their axons cross to the contralateral medial longitudinal fasciculus and make excitatory synapses with the contralateral medial rectus motor neurones (Carpenter and McMasters, 1963; Carpenter et al., 1963; Baker and Highstein, 1975). Robinson (1970 has proposed that anterior internuclear ophthalmoplegia is caused by a lesion in the medial longitudinal fasciculus which affects both the inhibitory input to the ipsilateral medial rectus neurones and the excitatory input to the contralateral medial rectus. The lack of inhibition to the ipsilateral medial rectus means that abduction in this eye should be slower than normal and this has been demon- 
strated physiologically by Loeffler et al. (1966) and behaviourally by Bird and Leech (1976).

If it is assumed that a lesion can affect only the inhibitory pathway to the ipsilateral medial rectus, only abduction in that eye will be slowed, as in many of our subjects. This may be one mechanism of posterior internuclear ophthalmoplegia.

Finally, an explanation may be offered for the fact that centring abduction is often weak when decentring appears normal. During adduction all abducens neurones are inhibited (Fuchs and Luschei, 1970). In contrast, during abduction, medial rectus motor neurones may continue firing (Robinson, 1970). The number and firing rates of oculomotor neurones increase in proportion to eye displacement in the 'on' direction (Schiller, 1970). Hence if the eye begins to abduct from a nasal position in which fewest motor units are recruited, and inhibition is reduced on the antagonist motor neurones, the abduction will be reduced in its effectiveness.

\section{Conclusion}

The simple geometry of the eye and the ease of electro-oculographic recording enable precise quantitative descriptions of oculomotor function to be given. As a consequence, eye movement tests are very sensitive indicators of neurological diseases.

It has been demonstrated that when eye movement tests are carried out with the head placed in eccentric positions, disorders of eye movement are recorded which are not apparent on conventional testing. In particular, disorders of conjugate gaze occur. These 'latent' defects of eye movement appear because, when the head is turned, there are differential compression and stretching effects on the vertebral arteries which may lead to local or generalised vertebro-basilar ischaemia if the vasculature is inherently defective.

Head turning reveals disorders of conjugate gaze, particularly in patients with suspected lesions of the low brain stem and cerebellum. The test also increases the incidence of dissociation of gaze in patients with multiple sclerosis. In this disease, two hypotheses to explain the effect of head turning are tenable. Either local restrictions of blood supply exacerbate faulty transmission in diseased nervous tissue or the mechanical stretching and compression on nerves, which result directly from twisting the brain stem, adversely affect the mechanisms of transmission.

The most common finding in patients with multiple sclerosis is a weakness of abduction. This can be explained by the hypothesis that there is a lesion of the inhibitory pathway to the ipsilateral medial rectus muscle (antagonist of the abduction). This hypothesis unifies the explanations of posterior and anterior internuclear ophthalmoplegia and distinguishes them as functional rather than anatomical entities.

The head turning test reveals defects of eye movement in otherwise healthy young people and thus may be a sensitive indicator of inherent skeletal and vascular defects.

\section{References}

Baker, R., and Highstein, S. M. (1975). Physiological identification of interneurons and motorneurons in the abducens nucleus. Brain Research, 91, 292-298.

Biemond, A. (1951). Thrombosis of the basilar artery and the vascularization of the brain stem. Brain, 74, 300-317.

Biemond, A., and De Jong, J. M. B. (1969). On cervical nystagmus and related disorders. Brain, 92, 437-458.

Bird, C. A., and Leech, J. (1976). Internuclear ophthalmoplegia: An electro-oculographic study of peak angular saccadic velocities. British Journal of Ophthalmology, 60, 645-651.

Bos, J. H. (1962). On Vestibular Nystagmus without Causative Endolymph Displacement. Drukerij Cloeck en Moedigh NV: Amsterdam.

Bos, J. H., and Philipszoon, A. J. (1963). Some forms of nystagmus provoked by stimuli other than accelerations. Practica Oto-Rhino-Laryngologica (Basel), 25, 108-118.

Carpenter, M. B., and McMasters, R. E. (1963). Disturbances of conjugate horizontal eye movement in the monkey. II. Physiological effects and anatomical degeneration resulting from lesions of the medial longitudinal fasciculus. Archives of Neurology (Chicago), 8, 347-368.

Carpenter, M. B., McMasters, R. E., and Hanna, G. R. (1963). Disturbances of conjugate horizontal eye movements in the monkey. I. Physiological effects and anatomical degeneration resulting from lesions of the abducens nucleus and nerve. Archives of Neurology (Chicago), 8, 231-247.

Cogan, D. G. (1968). Down beat nystagmus. Archives of Ophthalmology, 80, 757-768.

Cohen, L. A. (1961). Role of eye and neck proprioceptive mechanisms in body orientation and motor coordination. Journal of Neurophysiology, 24, 1-11.

Ford, F. R. (1952). Syncope, vertigo and disturbances of vision resulting from intermittent obstruction of the vertebral arteries due to defect in the oclontoid process and excessive mobility of the second cervical vertebra. Bulletin of the Johns Hopkins Hospital, 91, 168-173.

Fuchs, A. F., and Luschei, E. S. (1970). Firing patterns of abducens neurones of alert monkeys in relationship to horizontal eye movements. Journal of Neurophysiology, 33, 382-392. 
Gray, L. P. (1956). Extra-labyrinthine vertigo due to cervical muscle lesions. Journal of Laryngology, 70, 352-360.

Kleyn, A. De (1939). Some remarks on vestibular nystagmus. Confinia Neurologica, 11, 257-292.

Kleyn, A. De, and Nieuwenhuyse, P. (1927). Schwindelanfälle und nystagmus bei einer bestiminten stellung des kopfes. Acta Otolaryngologica, 11, 155157.

Loeffler, J. D., Hoyt, W. F., and Slatt, B. (1966). Motor excitation and inhibition in internuclear palsy. Archives of Neurology (Chicago), 15, 664-671.

Longet, F. A. (1845). Physiologie expérimentale sur les troubles qui surviennent dans l'équilibration, la station et la locomotion des animaux, après la section des parties molles de la nuque. Gazette Médicale de Paris, 13, 565-567.

McAlpine, D., Lumsden, C. E., and Acheson, E. D. (1972). Multiple Sclerosis: a reappraisal, pp. 243244. Churchill Livingstone: Edinburgh and London.

Maeda, M., Shimazu, H., and Shinoda, Y. (1972). Nature of synaptic events in cat abducens and motorneurones at slow and quick phases of vestibular nystagmus. Journal of Neurophysiology, 35, 279-296.

Magnus, R. (1924). Köperstellung. Springer Verlag: Berlin.

Meiry, J. L. (1971). Vestibular and proprioceptive stabilization of eye movements. In The Control of Eye Movements. Edited by P. Bach-y-Rita. Academic Press: New York and London.
Pola, J., and Robinson, D. A. (1976). An explanation of eye movements seen in internuclear ophthalmoplegia. Archives of Neurology (Chicago), 33, 447452.

Rasminsky, M. (1973). The effects of temperature on conduction in demyelinated single nerve fibers. Archives of Neurology (Chicago), 28, 287-292.

Robinson, D. A. (1970). Oculomotor unit behavior in the monkey. Journal of Neurophysiology, 33, 393404.

Schiller, P. H. (1970). The discharge characteristics of single units in the oculomotor and abducens nuclei of the unanesthetized monkey. Experimental Brain Research, 10, 347-362.

Sheehan, S., Bauer, R. B., and Meyer, J. S. (1960). Vertebral artery compression in cervical spondylosis. Neurology (Minneapolis), 10, 968-986.

Smith, K. U., Schmidt, T., and Putz, V. (1970). Binocular co-ordination. American Journal of $\mathrm{Op}$ tometry, 47, 679-689.

Taylor, A. R., and Byrnes, D. P. (1974). Foramen magnum and high cervical cord compression. Brain, 97, 473-480.

Tissington, W. F. T., and Bammer, H. G. (1957). Syndrome of vertebral artery compression. Neurology (Minneapolis), 7, 331-340.

Vossius, G. (1960). Das system der augenbewegung. Zeitschrift für Biologie, 112, 27-57.

Weber, R. B., and Daroff, R. B. (1971). The metrics of horizontal saccadic eye movements in normal humans. Vision Research, 11, 921-928. 